\title{
Efectos de los ejercicios periorales e intraorales en niños prematuros para estimular los reflejos orofaciales
}

Débora Elizabeth Escobar Ruíz ${ }^{1}$

DOI del artículo: 10.36631/Arrupe.2021.06.03

Fecha de recepción: 26/10/2021

Fecha de aceptación: 28/10/2021

\section{Resumen}

Han sido varios estudios realizados para la estimulación de los reflejos orofaciales y sus alteraciones, en el rango de edades y géneros de una población de neonatos prematuros, pero a nivel del departamento de Quetzaltenango no existe un estudio que describa las alteraciones en los reflejos orofaciales en neonatos prematuros; de manera que surge la necesidad de contextualizarlos a partir de realizar un estudio cuasi experimental cuyo objetivo fue aplicar un tratamiento a un solo grupo de individuos, sin tener que recurrir a un grupo control. A través de ello se pudo observar el estado del paciente antes, durante y después de la estimulación orofacial en niños prematuros. Para la recolección de datos se empleó la evaluación sensorio motora oral del bebé, ya que es una herramienta válida para el diagnóstico de las disfunciones orales del bebé. El estudio se realizó durante los meses de junio a septiembre de 2018, en la unidad de cuidados intermedios del Hospital Regional de Occidente, con la autorización de los padres; se concluye que la mayoría de los neonatos prematuros presentan una prevalencia en alteraciones orofaciales. Estos resultados permiten iniciar un estudio cuasi experimental donde se apliquen técnicas de fisioterapia, para que se puedan estimular los reflejos orofaciales que se encuentran alterados por la prematurez.

Palabras clave: ejercicios periorales e intraorales, prematurez, reflejos orofaciales

\begin{abstract}
Several studies have been carried out for the stimulation of orofacial reflexes and their alterations, in a range of ages and genders of a population aimed at premature infants, but at the level of the department of Quetzaltenango there is no study that describes alterations in orofacial reflexes in premature neonates; Thus, the
\end{abstract}

${ }^{1}$ Licenciada en Fisioterapia. Universidad Rafael Landívar, Campus de Quetzaltenango. 
need to contextualize them arises from a quasi-experimental study whose objective is to apply a treatment to a single group of individuals, without having to resort to a control group. Through this, the state of the patient can be observed before, during and after orofacial stimulation in premature children. For data collection, the baby's oral sensory motor evaluation was used, since it is a valid tool for the diagnosis of the baby's oral dysfunctions. The study was carried out during the months of June to September 2018 in the intermediate care unit of the Hospital Regional de Occidente, with the authorization of the parents; It is concluded that the majority of preterm infants present a prevalence of orofacial alterations. These results allow starting a quasi-experimental study where physiotherapy techniques are applied, so that orofacial reflexes that are altered by prematurity can be stimulated.

Key words: Perioral and intraoral exercises, prematurity, orofacial reflexes

\section{Introducción}

Mallma, en el estudio titulado «Incremento de la frecuencia de la terapia orofacial sobre la evolución de las fases de la succión nutritiva en neonatos pretérmino tardío», cuyo objetivo fue demostrar, que al incremento de la frecuencia de la terapia orofacial es mayor la evolución de las fases de la succión nutritiva, en neonatos pretérmino tardío. La conclusión obtenida en el estudio fue que el incremento de la frecuencia de la terapia orofacial, mejora la evolución de las fases de la succión nutritiva, en lo que respecta a las fases de expresiónsucción y deglución, pero no en la fase de respiración. A la vez recomienda la utilización de la terapia orofacial (1). 
Riquelme, en el estudio titulado «Estimulación de las aptitudes motrices bucofaciales en niños pretérmino», cuyo objetivo fue comprobar si la estimulación de los reflejos orofaciales innatos, disminuye el tiempo que necesita el niño prematuro para adquirir una succión nutritiva, con ejercicios de relajación global y facial y estimulación perioral e intraorales, movimientos de mejillas, mandíbula y lengua. La conclusión obtenida en el estudio fue que muchos autores han mostrado la eficacia de la estimulación de la succión no nutritiva, con programas de estimulación oral, a la vez recomienda valorar los resultados en una muestra mayor e introducir medidas biomecánicas en la evaluación (2).

Salcedo et al, en el estudio titulado «Retraso de la adquisición de la succióndeglución- respiración en el pretérmino; efectos de una estimulación precoz», cuyo objetivo fue analizar la transición de la nutrición enteral a la oral, la comorbilidad asociada con su retraso y el impacto de una estimulación precoz de la succión, en una cohorte de prematuros. La conclusión obtenida en el estudio fue que el estímulo precoz de la succión, en el paciente pretérmino, parece facilitar la nutrición oral completa de manera precoz; asociándose a una disminución en la estancia hospitalaria y a una mejora en la ganancia ponderal media diaria (3).

Según la OMS, en el año 2018, se estima que cada año nacen alrededor de 15 millones de niños prematuros y cada día esta cifra aumenta. En los 184 países estudiados, la tasa de nacimientos se promedia entre el $5 \%$ y el $18 \%$ de los recién nacidos, siendo la primera causa de muerte en los niños menores de 5 años. En Guatemala, el sistema de información gerencial en salud reportó 394817 partos, de los cuales 90413 fueron neonatos prematuros o con bajo peso al nacer.

Un bebé prematuro se encuentra en desventaja con un bebé a término, pues a pesar de los avances de la tecnología ninguno 
de los métodos innovadores se compara al medio intrauterino normal.

\section{Fundamentos teóricos científicos}

Los reflejos orofaciales son importantes para realizar la actividad de alimentación, al momento del nacimiento, en muchos de los casos se encuentran ausentes o debilitados en los neonatos prematuros o a término; con bajo peso al nacer por la falta de madurez intrauterina, debido a que el período de gestación no llega a concluirse en el tiempo predeterminado. Por lo tanto, en estos casos se recurre a la colocación de sonda orogástrica, ya que el neonato, por sí solo, no puede realizar la succión del instrumento artificial o del pezón de la madre; a través de este método se podrán llenar los requerimientos nutricionales del bebé, hasta que pueda llegar al peso ideal y poder retirar dicha sonda.

Los ejercicios periorales e intraorales son efectivos, para su realización se requiere un protocolo de tratamiento estructurado, así como un conocimiento técnico para su aplicación. Al momento de diseñar un protocolo de tratamiento con ejercicios orofaciales se crea una opción nueva, práctica y segura para la población que necesita de estos ejercicios, con la finalidad de estimular y fortalecer la musculatura orofacial, por lo cual se promueve que el neonato pueda realizar los reflejos orofaciales por sí solo.

\section{Ejercicios periorales e intraorales}

Son los ejercicios que intervienen en las alteraciones de la alimentación y en el lenguaje, tanto en adultos como niños; engloba la estimulación sensorial y cognitiva y favorece la intencionalidad del entorno (4).

Esta estimulación agrupa una serie de ejercicios dirigidos a intervenir en problemas presentes en los neonatos prematuros, pues abarcan el proceso de succión, deglución y respiración que favorecen las actividades alimentarias, con el fin de estimular y fortalecer la musculatura orofacial del individuo y, por tanto, el desarrollo dentro de su entorno.

\section{Objetivos}

a) Permitir la regulación del tono muscular.

b) Promover un equilibrio en las funciones del complejo orofacial.

c) Integración del complejo orofacial.

d) Controlar y permitir movimientos, así como también posturas linguales.

e) Permitir el desarrollo de la comunicación verbal y no verbal. 


\section{Aplicación de la terapia de regulación orofacial}

Un aspecto importante para la aplicación de la terapia orofacial es una correcta y adecuada postura corporal; se comienza con el cambio de las compensaciones corporales que pueda presentar el individuo y el control del tono muscular (11).

A través de una adecuada alineación corporal se estimula al neonato de forma positiva, haciéndole conciencia de cada una de las partes de su cuerpo. Se tendrá cuidado del tono muscular que presente, ya que se debe evitar el aumento o disminución del mismo, pues podría generar dificultad al momento de desarrollar el tratamiento.

\section{Realización del tratamiento}

La preparación para realizar las técnicas y desarrollar el tratamiento son: contacto manual, deslizamientos, presión, tracción y vibración.
Antes de comenzar con la preparación es importante colocarlo en una postura adecuada, el neonato debe estar en alerta y tranquilo, mejor si tiene hambre. Se coloca en brazos, ligeramente flexionado, cabeza, cuello, cintura escapular alineados, siempre con el cuidado de la zona cervical para que no lleve la cabeza hacia atrás en extensión. Durante todo el proceso se debe observar el aspecto y el tono muscular del recién nacido, como también su comportamiento ante la fase de succión.

Por medio de este procedimiento se deben evaluar los reflejos orales, estructuras orofaciales y la coordinación entre succión, deglución y respiración. Un bebé podrá tener respuestas atípicas a diversos ítem evaluados, difícilmente tendrá una sola o todas ellas. Según el tipo de respuestas atípicas, que presente el neonato, podrá dar pistas sobre la mejor forma de proceder para reconducir la situación.
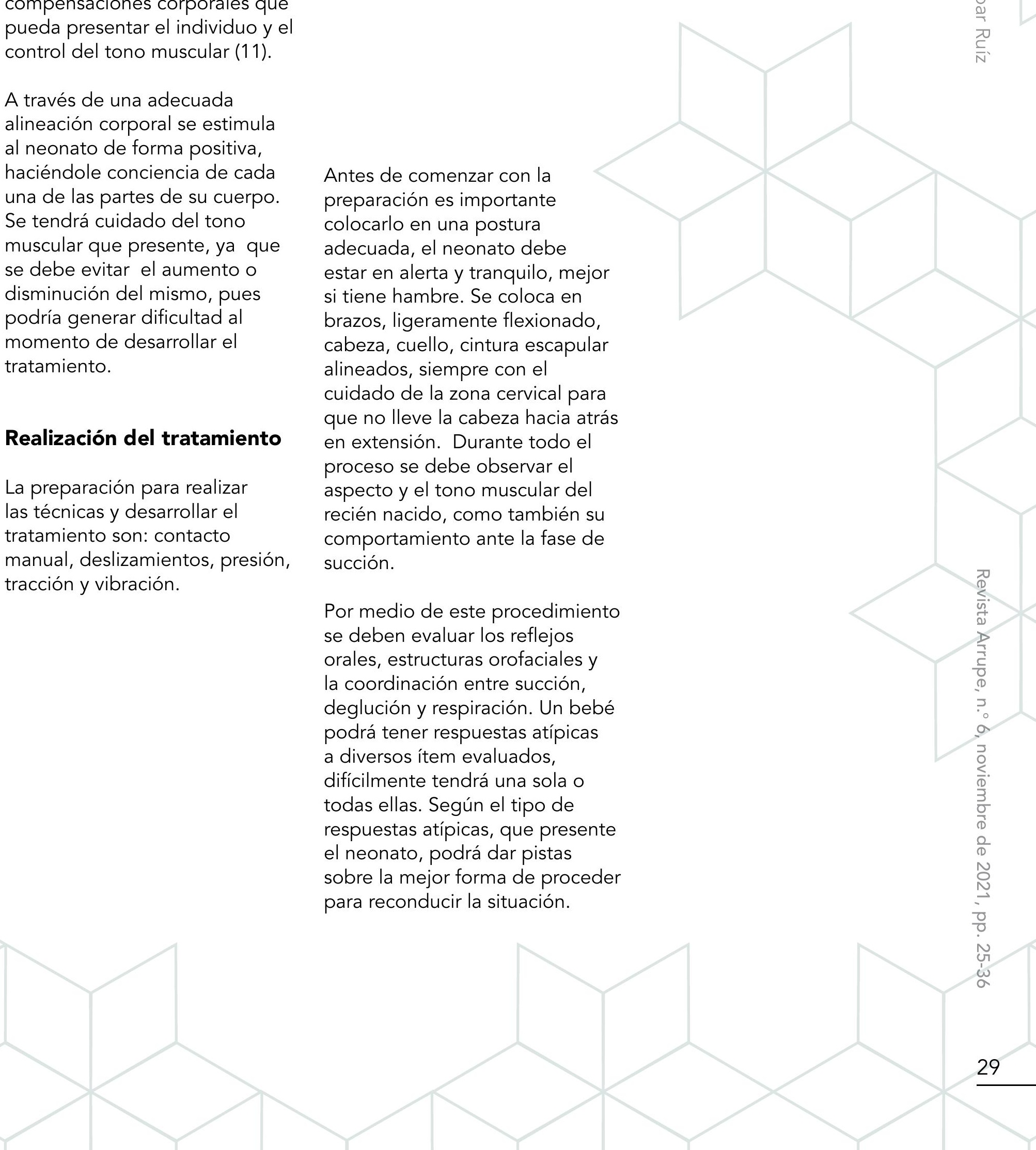


\section{a) Recursos técnicos}

- Contacto manual: la superficie de la mano con la que se va a trabajar, debe ser firme, a mano llena; no causar dolor y evitar por completo zonas que estén con heridas abiertas o sensibles por alguna patología.

- Deslizamiento: desplazamiento de la mano firme en determinada dirección y sentido, según el área que se va a trabajar.

- Tracción: separación de las carillas de una articulación. Al momento de efectuar tracción se debe tomar en cuenta el alineamiento corporal o del segmento a tratar.

- Vibración: son ondas producidas por una contracción isométrica. Para realizar de una forma correcta la vibración se debe tener al paciente en una adecuada posición corporal, no será funcional si no está alineado correctamente.

- Presión: fuerza a utilizar en las zonas orofaciales a trabajar (4). 


\begin{tabular}{|c|c|}
\hline \multicolumn{2}{|c|}{ Estimulación perioral } \\
\hline Descripción & Gráfica \\
\hline $\begin{array}{l}\text { a. Estimulación de carrillos: } \\
\text { se realiza barrido en carrillos desde } \\
\text { la articulación temporomandibular hacia } \\
\text { la comisura labial, con una presión media. } \\
\text { b. Objetivo: estimular el reflejo de búsqueda. } \\
\text { c. Repeticiones: cinco estimulaciones de } \\
\text { cada lado. }\end{array}$ & \\
\hline $\begin{array}{l}\text { a. Digitopresión: con el dedo índice y pulgar, } \\
\text { ejercer presión media de manera circular en } \\
\text { la zona de carrillos, desde la articulación } \\
\text { temporomandibular hacia la comisura. } \\
\text { b. Objetivo: estimular el reflejo de búsqueda } \\
\text { y apertura bucal. } \\
\text { c. Repeticiones: cinco estimulaciones en } \\
\text { cada lado. }\end{array}$ & \\
\hline $\begin{array}{l}\text { a. Digitopresión labial: con el dedo índice } \\
\text { trazar círculos alrededor de la musculatura } \\
\text { labial, con presión media. } \\
\text { b. Objetivo: estimular la musculatura labial, } \\
\text { para lograr que el neonato se dirija hacia el } \\
\text { estímulo en los cuatro puntos cardinales. } \\
\text { c. Repeticiones: cinco veces. }\end{array}$ & \\
\hline $\begin{array}{l}\text { a. Amasamiento digital: entre los dedos índice } \\
\text { y pulgar, protruir el labio inferior y el superior } \\
\text { y alternar de forma rápida el movimiento, pero } \\
\text { suave para no causar molestias. } \\
\text { b. Objetivo: comenzar a estimular el movimiento } \\
\text { de succión. } \\
\text { c. Repeticiones: diez veces. }\end{array}$ & \\
\hline $\begin{array}{l}\text { a. Masaje transversal con el dedo índice } \\
\text { sobre los labios: pasar el dedo índice } \\
\text { rápidamente por el labio superior; } \\
\text { alternar con el labio inferior de arriba hacia abajo. } \\
\text { b. Objetivo: preparar al neonato para la apertura } \\
\text { bucal. } \\
\text { c. Repeticiones: diez veces. }\end{array}$ & \\
\hline
\end{tabular}

Estimulación perioral

Descripción
a. Apertura bucal: con el dedo pulgar sobre la
barbilla del neonato se estimula con un
movimiento hacia abajo, en dirección al pecho,
con el fin de facilitar la apertura labial. Se introduce
el dedo índice y sobre el paladar se masajea
suavemente; se realiza encima de la configuración
del paladar, hacia un lado y hacia el otro.
b. Objetivo: propiciar la apertura bucal y estimular
el inicio de la succión.

c. Repeticiones: diez veces.

a. Estimulación del reflejo de extrusión: masajear suavemente sobre la lengua en dirección longitudinal, de derecha a izquierda, de forma sutil y rítmica, sin provocar náusea.

b. Objetivo: promover el reflejo de succión para que el neonato reconozca el estímulo y reciba el biberón o el pezón de la madre.

c. Repeticiones: diez veces.

a. Estimulación del reflejo de succión: en línea media sobre la lengua, colocar el dedo índice y realizar el movimiento de retracción y extensión, para activar el reflejo de succión.

b. Objetivo: activación del reflejo de succión.

c. Repeticiones: dos series de cinco repeticiones, con el cuidado necesario para no provocar nauseas en el bebé.

a. Masaje para aumentar el grado de tensión bucal: con el dedo índice masajear suavemente carrillos internos hacia afuera.

b. Objetivo: contribuir a la estimulación y fortalecimiento de la succión, para la toma correcta del biberón o el pezón de la madre.

c. Repeticiones: cinco veces en cada lado. 


\section{Reflejos orofaciales}

Los reflejos son respuestas innatas, comportamientos espontáneos o reacciones a los estímulos endógenos o ambientales, que se suscitan durante la vida fetal y se observan en todos los recién nacidos a término sanos, al nacer. Los recién nacidos (RN) están equipados con pautas específicas que determinan su conducta. Algunas de ellas son las que denominan reflejos y se caracterizan por ser muy estereotipados, constituyen la respuesta a estímulos específicos.

Estos reflejos son evaluables y entregan información sobre el estado del sistema nervioso, ya que su ausencia, presencia o alteración indican el estado de maduración cerebral. Por otra parte, los reflejos básicos son usados por el RN para la sobrevivencia. La mayoría de estos son asimilados en la medida en que el neonato desarrolla más control de su cuerpo y ambiente, mientras que otros desaparecen.

Los reflejos orales garantizan la función de alimentación en el período postnatal inmediato y son la base sobre la cual emergerán respuestas similares, a nivel voluntario. Poseen un control nervioso, que permite su desencadenamiento; pueden dividirse en función de su relación con la alimentación, ya sea mediante la adquisición de esta (reflejos adaptativos) o como mecanismo de protección durante la misma (reflejos protectores) (5).

La falta de maduración durante el periodo intrauterino desencadena varias dificultades en el desarrollo del neonato, por lo tanto, es importante dar a conocer cada uno de los aspectos que pueden afectarlo durante los primeros meses de vida.

Durante el proceso de succión-deglución-respiración es indispensable observar el desenvolvimiento del neonato, desde el agarre del pezón, la succión de la leche materna hasta finalizar la alimentación; a través de ello se determina la postura, el agarre, tono muscular del área 
orofacial, fuerza y coordinación. Todo ello dará la pauta del estado del neonato y qué áreas están debilitadas e impiden la correcta alimentación durante los primeros meses de vida.

Los reflejos involucrados en la alimentación son fundamentales para la nutrición del neonato. Al iniciar la octava semana de gestación, se activa la succión nutritiva, llamándose así porque los movimientos no son constantes ni rítmicos; en la semana 34, él bebé entra en un período de maduración en el cual se activa la succión nutritiva acompañado de la fase de deglución, vitales para los primeros días de vida. No obstante, la maduración y desarrollo de estos reflejos se completan a los 5 días de vida.
Para que exista una correcta coordinación de la succióndeglución-respiración es de suma importancia, que una serie de estructuras periféricas y centrales, se encuentren desarrolladas e integradas, con la finalidad de guiar cada uno de los reflejos involucrados en el proceso de alimentación.

Se debe tomar en cuenta que hay múltiples factores que pueden debilitar estas funciones, por lo tanto, es importante atenderlos a tiempo y erradicarlos, para evitar enfermedades o secuelas en el futuro.

\section{Resultados y discusión}

Tabla 1

Reflejos orofaciales

\begin{tabular}{|l|c|c|}
\hline \multicolumn{2}{|c|}{ Prueba T para medias de dos muestras emparejadas } \\
\hline & Estadístico T & $\begin{array}{l}\text { Valor Crítico de T } \\
\text { (Dos colas) }\end{array}$ \\
\hline Reflejo de búsqueda & $\mathbf{7 . 9 6}$ & \\
\hline Reflejo de succión & 10.34 & \\
\hline Reflejo de deglución & $\mathbf{9 . 6 5}$ & \multirow{2}{*}{2.06} \\
\hline Reflejo de extrusión & $\mathbf{8 . 4 7}$ & \\
\hline Reflejo de nauseoso & $\mathbf{5 . 8 2}$ & \\
\hline Reflejo de tos & $\mathbf{4 . 7 9}$ & \\
\hline Reflejo de mordedura & $\mathbf{6 . 7 8}$ & \\
\hline
\end{tabular}

Fuente: elaboración propia con base en los resultados del trabajo de campo 2018.

En la tabla 1 se puede analizar que hay cambios significativos en cada uno de los reflejos evaluados. Por los resultados obtenidos se determina, que en la evaluación de reflejos los resultados del estadístico $t$, son mayores al valor crítico $T$ (dos colas) $=2.06$. Por lo que indica que el tratamiento fisioterapéutico es efectivo 


\section{Gráfica 1}

Medias de reflejos orofaciales

\section{Final |||||||| Intermedia |||||||| Inicial}

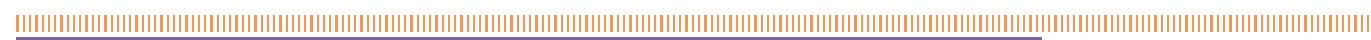 ||||||||||||||||||||||||||||||||||||||||||||||||||||||||||||||||||||||||||||||||||||||||||||||||||||||||||||||||||||||| \\ T) \\ Tos

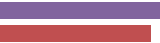 \\ Nauseoso

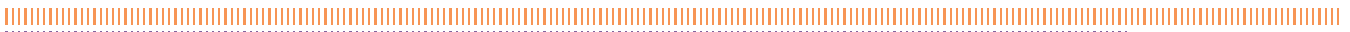

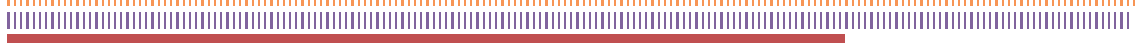 \\ Extrusión \\ |}

Deglución

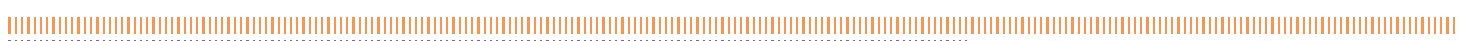
||||||||||||||||||||||||||||||||||||||||||||||||||||||||||||||||||||||||||||||||||||||||||||||||||||||||||||||||||||||||||||||||||||||||||||||||||||||||||||||||||||||||||||||

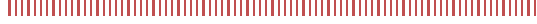

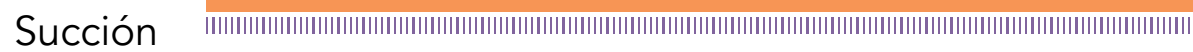

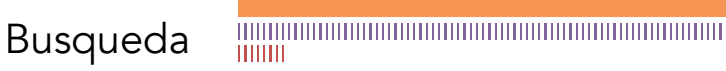
0
0.75
1.5
2.25

Fuente: elaboración propia con base en los resultados del trabajo de campo 2018.

En la gráfica 1 se puede observar que existe un aumento significativo en los reflejos: Reflejo de búsqueda, se obtiene una mejoría de 1.64 grados. Reflejo de succión donde se alcanza una mejoría de 2.6 grados. Reflejo de deglución presenta como resultado 1.64 grados de mejoría. Reflejo de extrusión con una mejoría de 1.6 grados. Reflejo nauseoso con un resultado de 0.92 grados de mejoría. Reflejo de la tos con una mejoría de 0.48 grados. Reflejo de mordedura con un resultado de 1.28 grados de mejoría; afirma una efectividad de los ejercicios periorales e intraorales para estimular los reflejos orofaciales en niños prematuros.

Tabla 2

Evaluación alteraciones funcionales

\begin{tabular}{|l|c|c|}
\hline \multicolumn{2}{|c|}{ Prueba T para medias de dos muestras emparejadas } \\
\hline & Estadístico T & $\begin{array}{l}\text { Valor Crítico de T } \\
\text { (Dos colas) }\end{array}$ \\
\hline Grado de tensión & $\mathbf{7 . 6 9}$ & \multirow{2}{*}{2.06} \\
\hline Dirección del movimiento & $\mathbf{5 . 2 6}$ & \\
\hline Movimientos & $\mathbf{5 . 8 4}$ & \\
\hline
\end{tabular}

Fuente: elaboración propia con base en los resultados del trabajo de campo 2018. 
En la tabla 2 podemos observar que existe un aumento significativo en evaluación de alteraciones funcionales, los resultados del estadístico t son mayores al valor crítico $\mathrm{T}$ (dos colas $)=2.06$. Por lo que indica que el tratamiento fisioterapéutico es efectivo.

\section{Gráfica 2}

Medias de alteraciones funcionales.

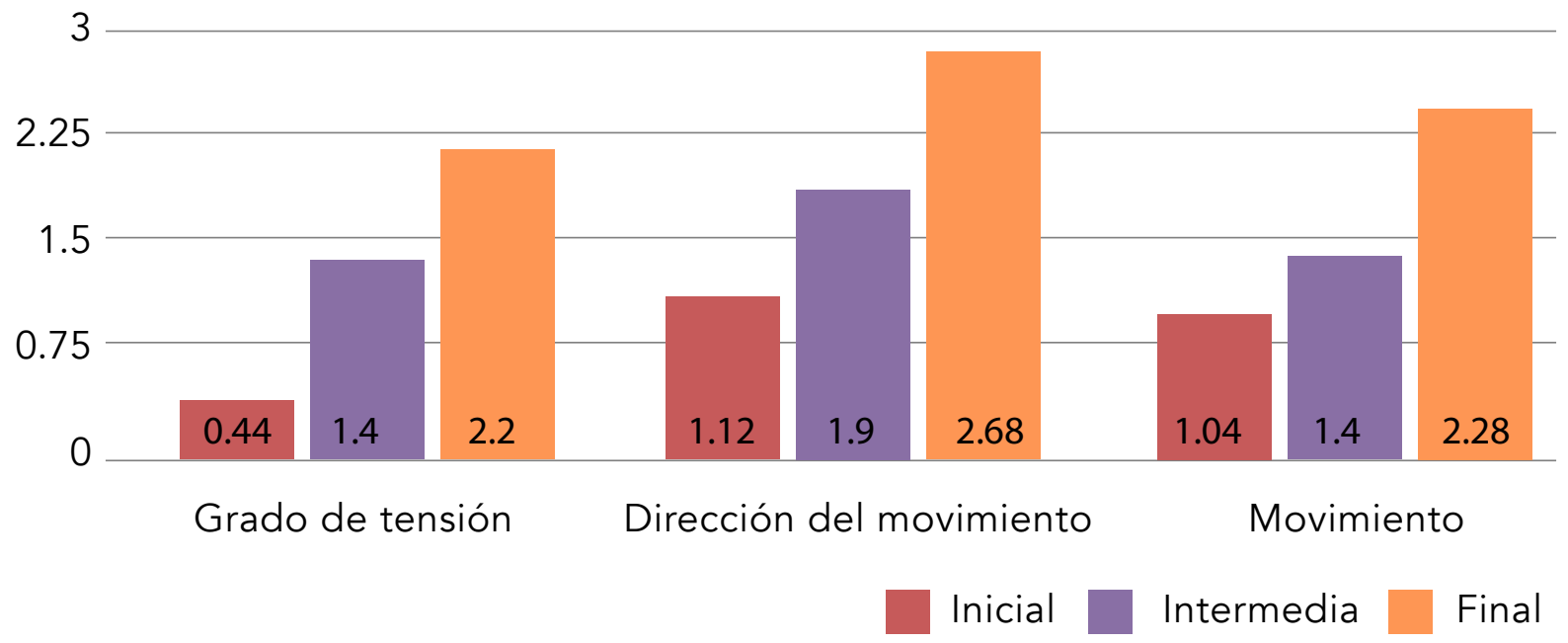

Fuente: elaboración propia con base en los resultados del trabajo de campo

En la gráfica 2 podemos observar que existe un aumento significativo en cuanto a las alteraciones funcionales, que se pueden presentar en el proceso de succión-deglución: Grado de tensión con un resultado de 1.76 grados de mejoría. Dirección del movimiento con una mejoría de 1.56 grados. El movimiento da como resultado 1.24 grados de mejoría; se afirma, por lo tanto, la efectividad de los ejercicios periorales e intraorales para estimular los reflejos orofaciales en niños prematuros. 


\section{Conclusiones}

1. Se determinó el estado inicial sensorio motor oral de cada uno de los pacientes, por medio de la calificación del grado de evolución, presentado en cada uno de los reflejos involucrados en el proceso de alimentación.

2. Se pudo comprobar el aumento del reflejo de succión y de los demás reflejos involucrados en el proceso de alimentación, por medio de la aplicación de los ejercicios periorales, a través del estímulo en cada una de las áreas, lo que posibilita al bebe realizar los reflejos adecuadamente.

3. Se determinó que la aplicación de los ejercicios intraorales contribuye en la alimentación del neonato prematuro y permite, por tanto, una coordinación adecuada en el proceso de succióndeglución.

4. Se verificó que hubo una notable mejoría en el reflejo de succión y los demás reflejos involucrados en el proceso de alimentación, lo cual permitió el retiro de la sonda orogástrica.

5. Al ser evaluados los resultados finales del tratamiento se confirmó que la aplicación de ejercicios periorales e intraorales, posibilita una adecuada recuperación de la funcionalidad y mejora la actividad de alimentación, sin necesidad de volver a recurrir a la sonda orogástrica.

\section{Referencias}

1. Mallma Huamani, CY. Incremento de la frecuencia de la terapia orofacial sobre la evolución de las fases de la succión nutritiva en neonatos pretérmino tardío de la unidad de cuidados intermedios de neonatología. Cibertesis. Tesis, Universidad Nacional Mayor de San Marcos, 2012; p. 7. https://hdl.handle.net/20.500.12672/2887

2. Riquelme, A. Estimulación de las aptitudes motrices bucofaciales en niños pretérmino. Universidad de La Rioja, Área de Fisioterapia. España. 2007.

3. La Orden-Izquierdo, Salcedo-Lobato, E., Cuadrado-Pérez, I., et al. Retraso de la adquisición de la succión-degluciónrespiración en el pretérmino; efectos de una estimulación precoz. Nutrición Hospitalaria. 27(4), 2012:1120-1126. https://dx.doi.org/10.3305/nh.2012.27.4.5848

4. Luna, L. Intervención interdisciplinar desde el ámbito de la atención temprana, educación y sanidad del niño con alteraciones motoras o riesgo de padecerlas (prematuros). Universidad de Murcia. España. 2012. Pág. 1-6.

5. Asociación Española de Pediatría. Manual de lactancia materna de la teoría a la práctica. Madrid España: Editorial Panamericana, 2013. Pág. 217.

6. Sánchez. M. Programas de intervención para la disminución de la morbilidad en el prematurao y prevención de las secuelas en el el desarrollo. En: Atención del neonato prematuro en la UCIN. México DF: Editorial el Manual Moderno S.A de C.V, 2014;66-72. 